\title{
CORES OF EARLY-TYPE GALAXIES
}

\author{
John Kormendy ${ }^{1}$ \\ Dominion Astrophysical Observatory \\ Herzberg Institute of Astrophysics
}

\begin{abstract}
Systematic study of galaxy cores has become possible through improved understanding of seeing and the reduction of photometric errors by the use of CCDs. Many cores are well resolved in a photometry program with the Canada-FranceHawaii Telescope (median stellar FWHM $=0$ "' 80 ). Core profile shape correlates with galaxy luminosity $L$ : the brightest galaxies have isothermal profiles; fainter ellipticals and bulges have profiles that do not completely flatten inward into a core. This may be due to velocity anisotropies. Core parameters are correlated: more luminous galaxies have larger core radii and fainter central surface brightnesses. Large deviations suggest special events: Fornax A has too small and bright a core for its luminosity; it may be the remnant of a merger with a smaller galaxy. Core massto-light ratio $M / L \propto L^{0.2}$, as expected from the metallicity-luminosity relation. A kinematic search shows strong evidence for a central black hole in M31 and weaker evidence in M32 and NGC 3115. The nucleus of M31 rotates very rapidly but has an outer dispersion of only $107 \mathrm{~km} \mathrm{~s}^{-1}$. This implies that it is a disk; it may have formed from gas falling into the center. Rapid rotation and a central velocity dispersion $>241 \pm 7 \mathrm{~km} \mathrm{~s}^{-1}$ imply a central $M / L_{V} \gtrsim 20-35$. Velocity anisotropies are not a major uncertainty because of the rapid rotation. Therefore there is strong evidence for a nuclear point mass of $\sim 2 \times 10^{7} M_{\odot}$.
\end{abstract}

\section{INTRODUCTION}

Cores of elliptical galaxies are of interest for many reasons. The structure and stellar velocity distribution are easiest to understand in cores. Cores also have well-defined physical scales, the central surface brightness $\mu_{0}$, the core radius $r_{c}$ at which the surface brightness has fallen by a factor of two, and the central velocity dispersion $\sigma$. The systematic properties of these parameters can give insight into galaxy formation and evolution. Perhaps most interesting is the possibility that galaxy nuclei contain massive black holes that are the engines powering nuclear radio activity and quasars. A review of core structure based on photographic photometry is given in Kormendy (1982a). Since then, considerable progress has been made through major improvements in resolution and photometric accuracy.

1 Visiting Astronomer, Canada-France-Hawaii Telescope, operated by the National Research Council of Canada, the Centre National de la Recherche Scientifique of France, and the University of Hawaii. 


\section{TWO TECHNICAL BREAKTHROUGHS}

Two developments have allowed us to obtain reliable core photometry. First, Schweizer $(1979,1981)$ convinced us of the importance of seeing corrections, which formerly were made crudely or not at all. He pointed out that the tiny nuclei of M31 and M32 would not be resolved if they were observed as far away as the Virgo Cluster. Moreover, some apparent cores in Virgo were better described by seeingconvolved $r^{1 / 4}$ laws than by any isothermal or King (1966) model with a physical core (Fig. 1a). Schweizer showed further that if the central profile is as "cuspy", say, as an $r^{1 / 4}$ law, then seeing produces a fake core whose apparent radius $r_{c, a p p}$ is determined by the effective radius $r_{e}$ (Schweizer 1979, Fig. 4; Kormendy 1982a, Fig. 20). If the apparent core is little or no bigger than the radius predicted from $r_{e}$, we cannot be sure that it is resolved. It could be resolved, because seeing effects are relatively small for real cores. But the profile could be an $r^{1 / 4}$ law right to the center, or the galaxy could be hiding a nucleus like the one in M31. At that time, only M31 and the ellipticals NGC 4649, NGC 4472 and M87 were securely resolved. Schweizer argued that other galaxies might not have cores at all.

Therefore core parameters could only be derived if one assumed that galaxies had isothermal cores. Then seeing corrections were calculable and modest. Photometry by King (1978) and others was analyzed in this way by Kormendy $(1982 a, 1984)$, who found that $r_{c}, \mu_{0}$, and $\sigma$ correlate with each other and with galaxy luminosity $L$. However, the results were model-dependent: the seeing corrections would have been larger if the profiles were cuspy. Current CCD data are accurate enough for less model-dependent seeing corrections based on deconvolutions (Bendinelli et al. 1984, and references therein; Lauer 1985a, $b$ ). Nevertheless, we need the best possible seeing for accurate work on profile shapes and characteristic parameters, especially since small galaxies have tiny cores.
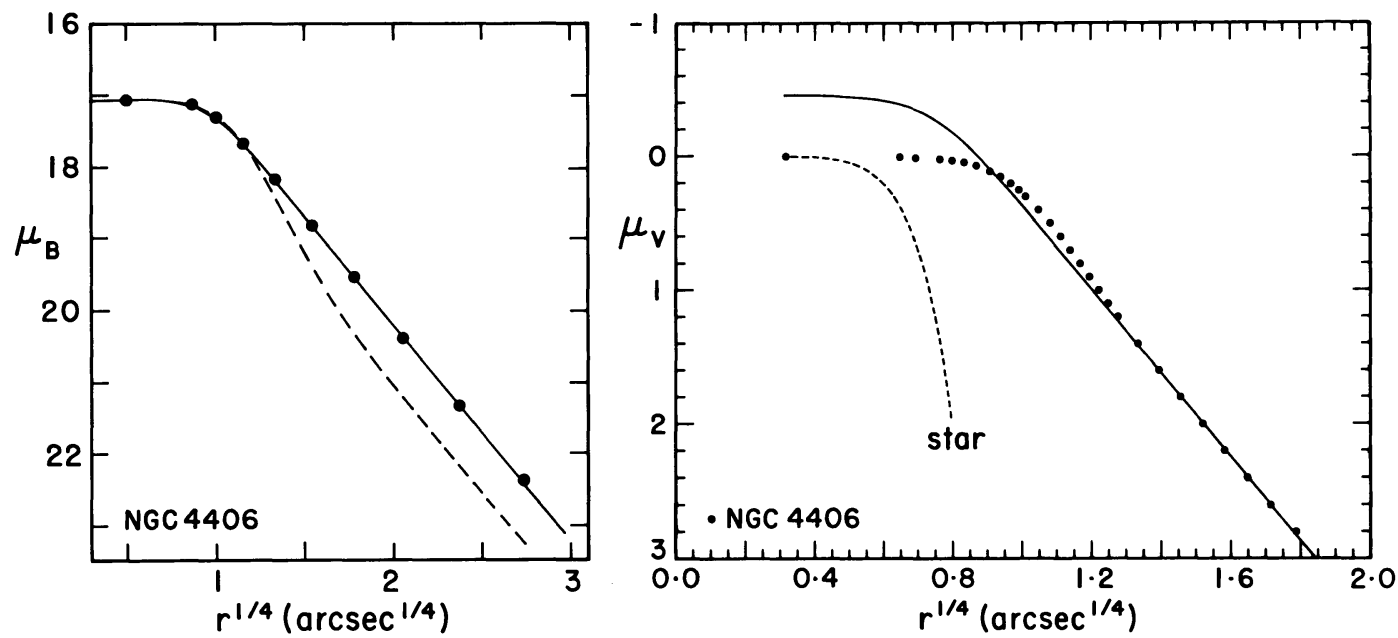

Fig. 1. - (a, left) Brightness profile of NGC 4406 (King 1978) compared with a seeing-convolved $r^{1 / 4}$ law (solid line) and a King model fitted at $r_{c}$ (dashed line) (Schweizer 1979). ( $b$, right) The profile of NGC 4406 measured in excellent seeing $\left.\sigma_{*}=0.21\right)$ is not consistent with a seeing-convolved $r^{1 / 4}$ law. 
The key to the present paper is the excellent seeing at the Canada-FranceHawaii Telescope. Most of the results discussed here were obtained in a photometry program carried out with an RCA CCD at the Cassegrain focus of the CFHT. The scale was $0 !^{\prime \prime 215}$ pixel $^{-1}$. Figure 2 shows the histogram of Gaussian seeing dispersion radii $\sigma_{*}$. It hardly overlaps with histograms for two well-known studies of cores which were themselves carried out at sites known for good seeing (Mount Wilson Observatory and Lick Observatory). The median $\sigma_{*}=0 ! 34$ is a factor of two better than in previous data. As a result, many cores are well resolved. Figure $1 b$ shows the improvement for NGC 4406. The profile is no longer consistent with a seeing-convolved $r^{1 / 4}$ law. The ratio $r_{c, a p p} / \sigma_{*}=8.4$; this implies that the galaxy is well resolved according to Schweizer's (1979) criterion. Almost all of the bright ellipticals in Virgo and even many that are several times farther away are this well resolved. Lauer $(1985 a, b)$ resolved several additional ellipticals and also found nearly isothermal cores.

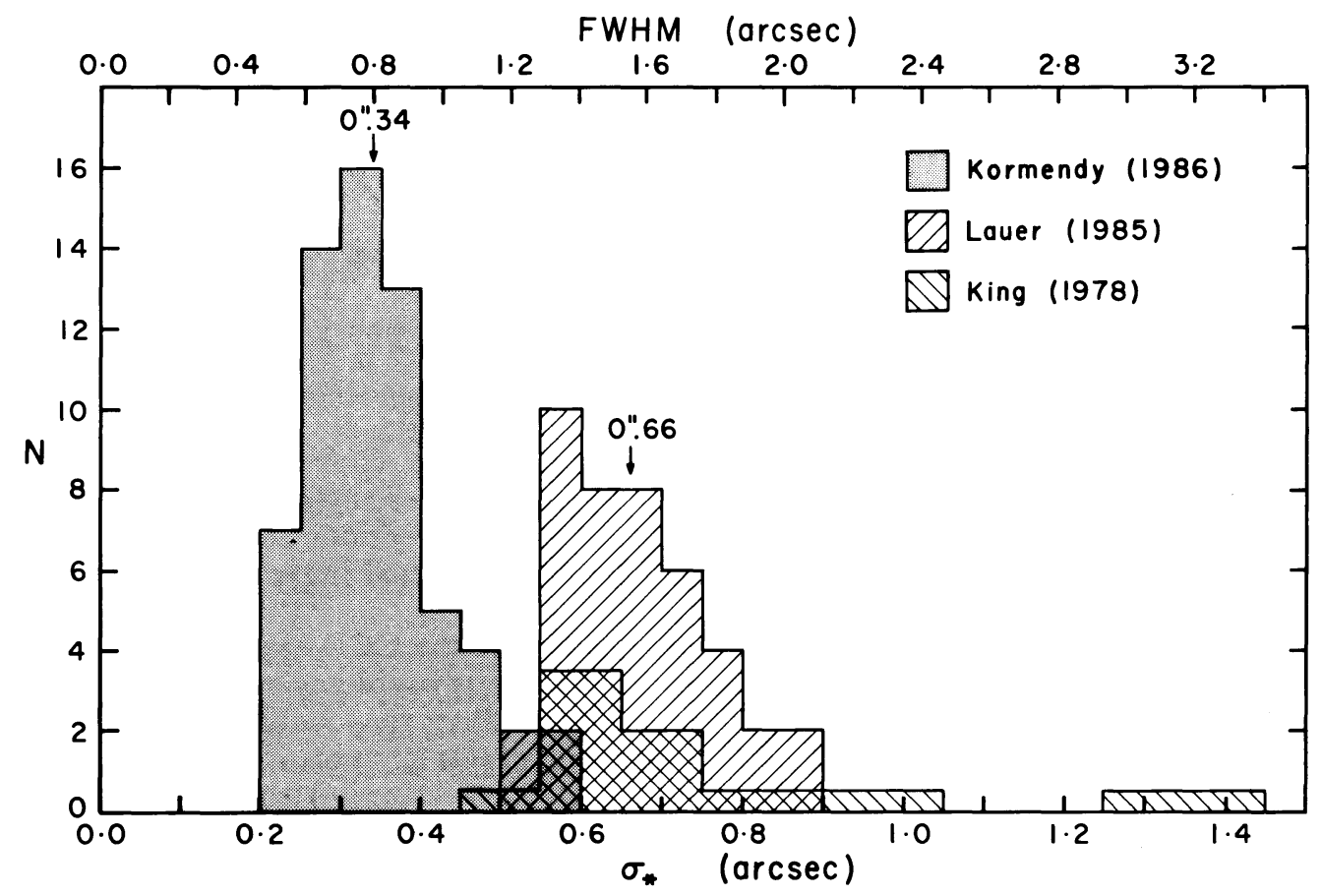

Fig. 2. - Histogram of seeing dispersion radii $\sigma_{*}$ for the present CFHT photometry and for two published studies of cores. The arrows indicate median values; they are equal for the King (1978) and Lauer (1985a) samples.

It now seems safe to assume that ellipticals generally have cores. Seeing corrections based on deconvolutions will eventually be made, but here I adopt a short-cut (Fig. 3) calculated by convolving a King model with $\log \left(r_{t} / r_{c}\right)=2.25$ and the point-spread function of the CFHT. It is convenient to parametrize the corrections in terms of $r_{c, a p p} / \sigma_{*}$. Galaxies with $r_{c, a p p} / \sigma_{*} \leq 3$ are poorly resolved; their seeing corrections still depend on the assumption that cores are nearly isothermal. In many cases the derived parameters are little better than limits. At the other extreme, galaxies with $r_{c, a p p} / \sigma_{*}>5$ are well resolved; the profile shape and core parameters are largely independent of assumptions. 


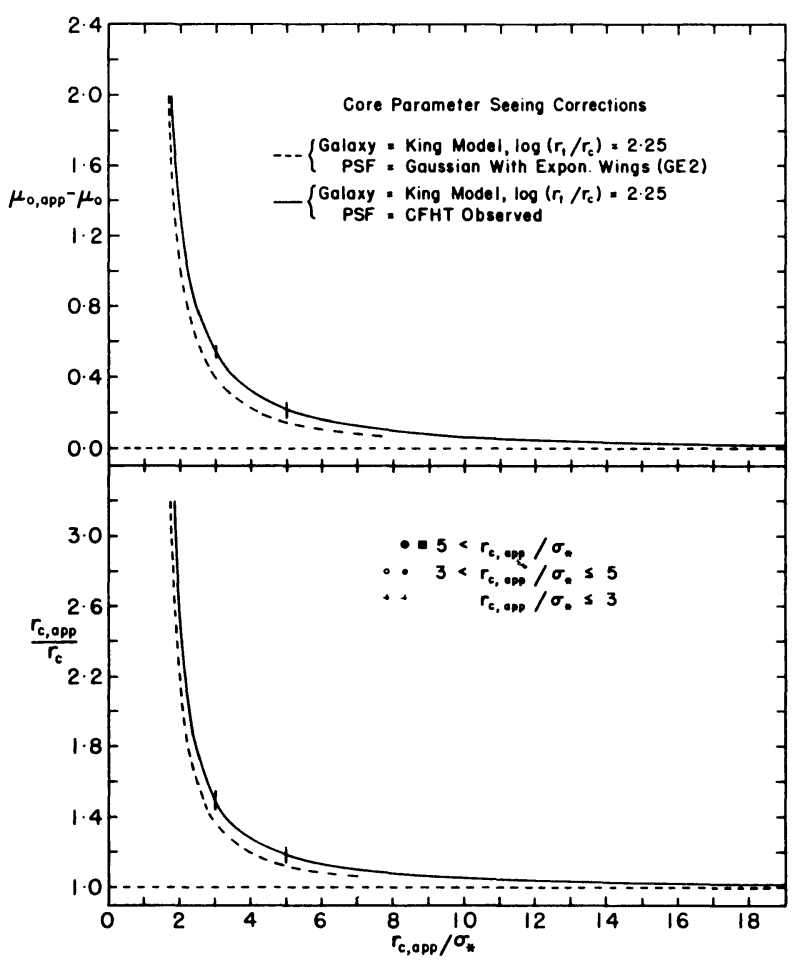

Fig. 3. - Seeing corrections (solid lines) used to convert apparent core parameters $r_{c, a p p}$ and $\mu_{0, a p p}$ to true values $r_{c}$ and $\mu_{0}$. The key shows how symbol sizes are used to indicate small, moderate and large corrections in Figure 6. The corrections derived by Schweizer (1981) are shown as dashed lines.

The second problem with core photometry before the CCD era was systematic errors. Lauer (1985a) has shown that there exist serious problems even with careful photographic photometry. Their cause is unclear. They may be due to problems with calibration or developer exhaustion, because fainter photographic photometry is more reliable. In contrast, my CFHT photometry agrees well with Lauer's seeingcorrected profiles but not with his raw data (e. g., Fig. 4). Similarly, our seeingcorrected core parameters agree well provided that we both resolved the galaxy. The mean ratio of core radii determined by Lauer and by me is $\left\langle r_{c L} / r_{c K}\right\rangle=0.99 \pm 0.01$ for 4 galaxies with $r_{c}>3^{\prime \prime}$ and Lauer resolution class I ("well resolved"); $1.04 \pm 0.03$ for all 8 galaxies with resolution class I; $1.24 \pm 0.11$ for 4 galaxies with resolution class II and 2.3 for 1 galaxy with resolution class III ("unresolved"). This shows (i) that the measurements agree well, (ii) that Lauer's seeing corrections are accurate when they are not too large, and (iii) that when his seeing corrections are large, they are underestimated. The same is probably true of my seeing corrections. In general, CCD photometry by various authors agrees to $\sim 0.1 \mathrm{mag} \operatorname{arcsec}^{-2}$ over many magnitudes. CCDs should be able to do better. However, this is accurate enough to allow large improvements over photographic work. The following discussion is based entirely on CCD data: those from the CFHT, Lauer's data for 4 galaxies of resolution class I that I have not observed, and Kent's (1983) data on M31. 


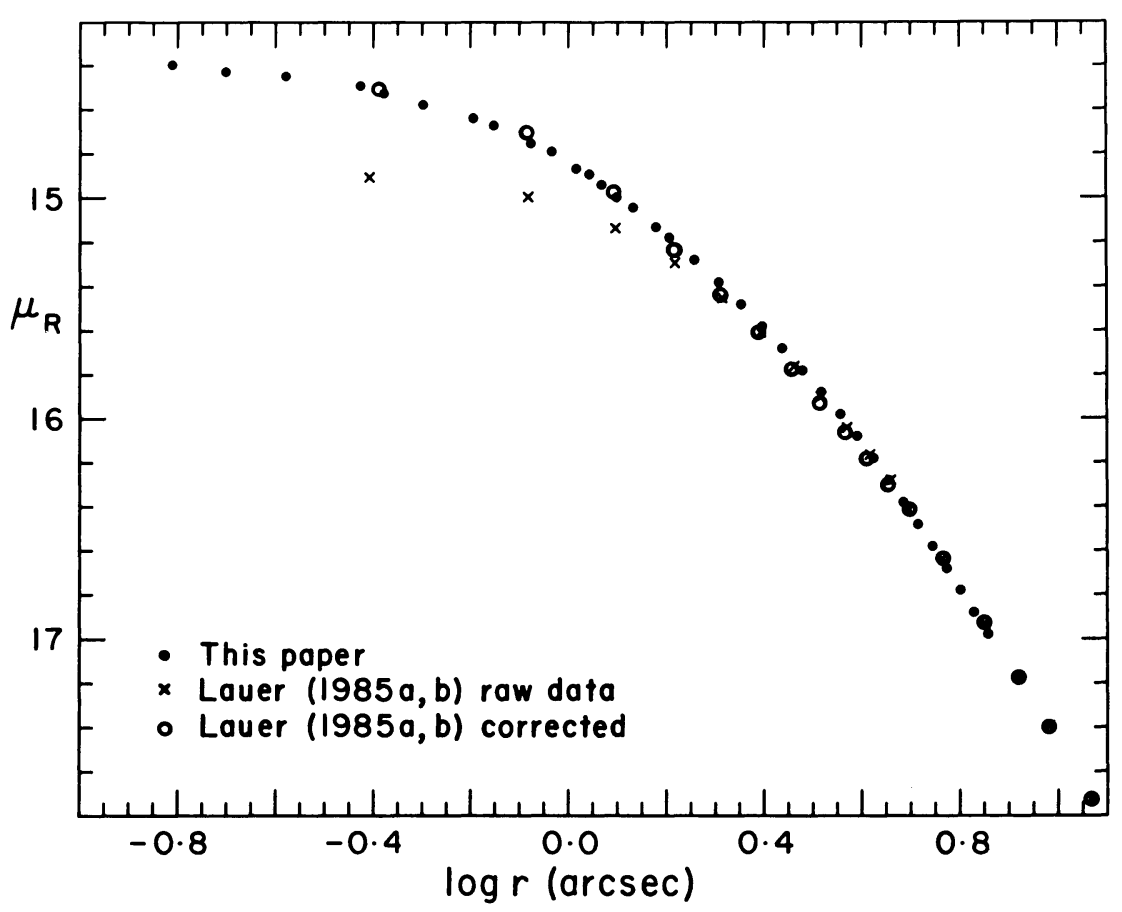

Fig. 4. - Comparison of uncorrected mean profile of NGC 3379 as measured with the CFHT $\left(\sigma_{*}=0 ! 21\right)$ with uncorrected and seeing-corrected profiles from Lauer $(1985 a, b)$.

\section{CORE PROFILE SHAPES}

The idea that core structure might be simple was suggested in part by early photographic photometry that showed isothermal central profiles. Eventually a number of cores were found that deviated from isothermals (King 1978; Kormendy $1982 a$ ), but this attracted little attention. Interest in velocity anisotropies was generated about 10 years ago by several nearly simultaneous events. It became clear on theoretical grounds (Binney 1976) that isotropy is not generally expected in ellipticals. In fact, they rotate slowly (Illingworth 1977); their shapes are determined not by rotation but by anisotropic velocity dispersions left over from galaxy formation (Binney 1978). The theoretical arguments applied equally well to cores; there was little more reason to expect them to be isotropic than ellipticals as a whole. The observational situation, however, was unclear. The second event was the claim that a black hole had been detected in the center of M87 based on an inward-rising dispersion profile (Sargent et al. 1978) and a brightness profile that did not flatten completely into a core at the center (Young et al. 1978). It was soon pointed out that anisotropies could explain these observations as easily as a black hole (Duncan and Wheeler 1980; Binney and Mamon 1982; Richstone and Tremaine 1985). Again it was not clear whether M87 was unusual or whether cores are generally not isothermal. 


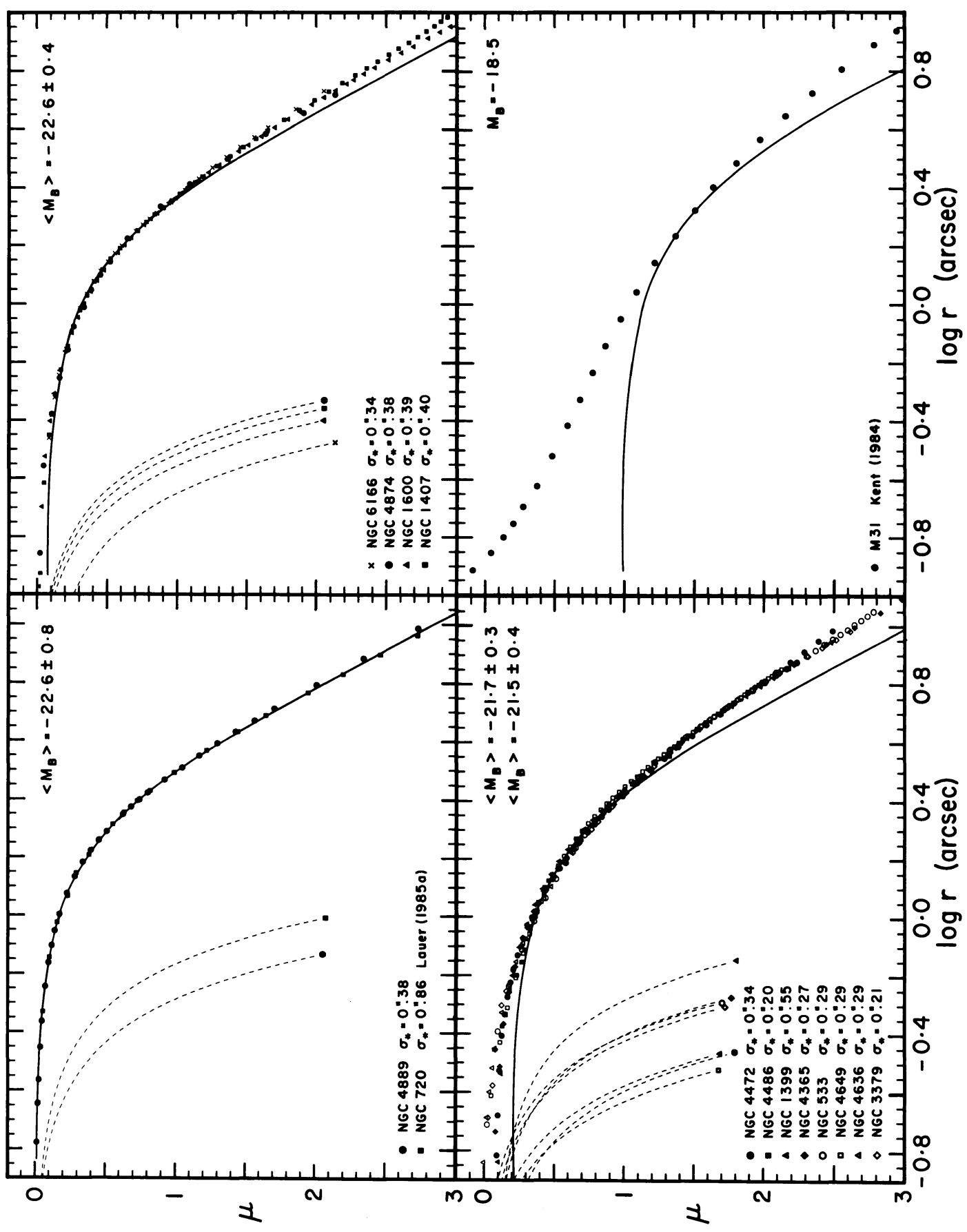


Fig. 5. - (Opposite) Composite mean-axis brightness profiles of well-resolved galaxies. The profiles have been shifted by arbitrary amounts in $\log r$ and $\mu$ (mag $\operatorname{arcsec}^{-2}$ ) to minimize the scatter. The M31 profile has been shifted to smaller radii by $\delta \log r=-1.2$. Each composite is compared with a projected isothermal model (solid lines). The bottom-left panel shows two groups of profiles; those plotted with open symbols are less isothermal near the center. Gaussian star profiles with the observed dispersions $\sigma_{*}$ (dashed lines) have been scaled with the galaxy profiles to illustrate the relative resolution. Each group of ellipticals is similarly well resolved, so the differences between them are not due to seeing. The mean absolute magnitude $\left\langle M_{B}>\right.$ of each group is also given.

Lauer $(1985 b)$ was the first to examine core profile shapes in a substantial sample of ellipticals using high-accuracy CCD data. He showed that virtually all cores have nonisothermal brightness profiles; M87 looks just like the rest. These results were extended by Kormendy (1985a) using high-resolution CCD photometry from the CFHT. As shown in Figure 5, core profile shape correlates weakly with galaxy luminosity. A few galaxies, usually the brightest first-ranked galaxies in clusters, have isothermal profiles. Fainter galaxies have profiles that do not flatten completely into a core. M31 is the faintest galaxy with a well-resolved core; it is even less isothermal than the ellipticals (Kent 1983). Other bulges are also less isothermal than ellipticals. In addition, many bulges have extra nuclei like that in M31, although the resolution is barely adequate to detect them even with the CFHT (Kormendy 1985a).

These data have not yet been modelled. For this we need rotation and velocity dispersion measurements with good resolution at $r \leq r_{c}$. Currently we have such data only for M31 (§5.2). Measurements are in progress with the CFHT. Kinematic evidence for anisotropy exists in several ellipticals; e. g., the core of NGC 1600 falls far below the "oblate line" describing isotropic oblate rotators in the $V_{\max } / \sigma-\epsilon$ diagram (Illingworth 1977). Like the overall shapes of ellipticals, the E3 shape of the core of NGC 1600 must be maintained by anisotropy. But despite the hints, we still have little systematic understanding of anisotropies in galaxy cores. Figure 5 suggests that there is considerable regularity from galaxy to galaxy, and that the anisotropies correlate with luminosity.

\section{CHARACTERISTIC PARAMETER CORRELATIONS}

\subsection{Correlations between $r_{c}, \mu_{0}, \sigma$, and $M_{B}$}

The observed correlations between core parameters of bulges and elliptical galaxies are scaling laws that provide important constraints for theories of galaxy formation. The best known is the Faber-Jackson (1976) observation that more luminous ellipticals have higher central velocity dispersions, $L \propto \sigma^{n}, n \simeq 4-6$. More recently, Kormendy $(1982 a, 1984)$ found that $r_{c}$ and $\mu_{0}$ are correlated with each other and with $\sigma$ and $M_{B}$. Similar relations were found by Lauer (1985b) from CCD photometry. Kormendy $(1985 b)$ has extended the $L$ range substantially and derived core parameters from high-resolution CFHT photometry. These results are updated in Figure 6. 


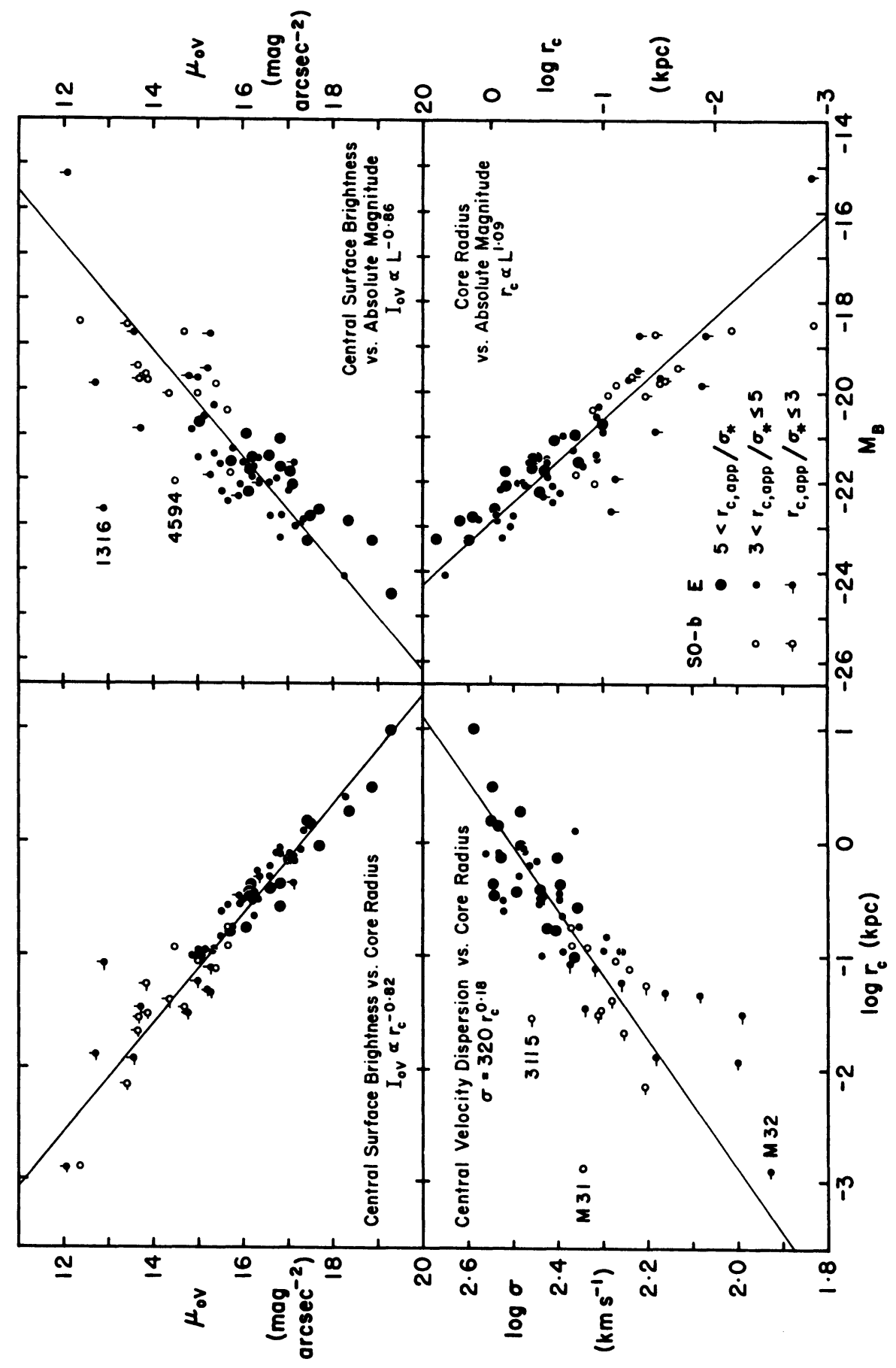


Fig. 6. - (Opposite) Core parameter correlations for bulges and elliptical galaxies. Distances are based on a Hubble constant of $50 \mathrm{~km} \mathrm{~s}^{-1} \mathrm{Mpc}^{-1}$. Open circles for bulges are derived using all of the observed profile, including any nucleus. Larger points imply better resolution of the core $(\S 2)$. The straight lines are leastsquares fits; deviant points (labelled with NGC numbers) are omitted.

More luminous galaxies have larger core radii $r_{c}$ and fainter central surface brightnesses $\mu_{O V}$. Also, $r_{c}$ and $\mu_{O V}$ correlate with $\sigma$, as expected from the FaberJackson relation. The least-squares fits are given in Figure 6; they are similar to ones derived in Kormendy $(\mathbf{1 9 8 5 b})$. It is interesting to note the variety of galaxies that are consistent with these relations. The high-luminosity end is defined by brightest cluster galaxies, including cDs like NGC 6166. The faintest galaxy plotted is M32; it is very poorly resolved, but its tiny core is normal for its low $L$. Between these extremes, radio galaxies (e. g., NGC 4874, M87, DA 240, and NGC 6251) and galaxies with X-ray cooling flows (e. g., NGC 4472, NGC 4636, and NGC 4649) are consistent with the correlations. Evidently they are not destroyed either by gas that falls into the center or by the engines that power nuclear activity. Nuclear black holes (if they exist) may be too small to grossly affect core structure: a galaxy with $M_{B}=-22$ has a core mass of $10^{10} M_{\odot}$. Bulges of disk galaxies also essentially satisfy the correlations. Some have small $r_{c}$ and bright $\mu_{O V}$ due to the presence of distinct nuclei (e. g., M31; Light et al. 1974). Apart from these nuclei, the cores of bulges and ellipticals are as similar as their global properties.

I mention here only two applications of the core parameter relations to the problem of galaxy formation. Carlberg (1987) shows that phase space densities in the cores of all but the largest ellipticals are much higher than those in galaxy disks. Since phase space density can only decrease during violent relaxation, this implies that ellipticals cannot have formed from stellar disks by dissipationless mergers. A second application is found in Kormendy $(1985 b, 1986)$. The core parameter relations of dwarf spheroidal galaxies are very different from those for ellipticals: there is a discontinuity between them of $>3$ orders of magnitude. Dwarf spheroidal galaxies fall on analogous parameter relations for the disks of spiral and irregular galaxies. This confirms a hypothesis that dwarf spheroidals are more closely related to dwarf spiral and irregular galaxies than to ellipticals. They may have evolved from dS + Im galaxies by losing their gas or converting it all into stars. This subject is reviewed in Kormendy (1986).

Much of the scatter in Figure 6 is real. Some is due to distance errors: the adoption of distances based on a Virgocentric flow field will reduce the scatter. Also, some of the scatter is due to a "second parameter" effect; this will be discussed when all of the data are reduced. Here I want to mention one galaxy that departs by large amounts from the correlations. NGC 1316 (Fornax A) has a nucleus that is much too small and bright for the high luminosity of the galaxy. Schweizer (1980) has suggested that NGC 1316 is a merger remnant, based on the existence of ripples in the light distribution, a possible tidal tail, and a central gas disk whose rotation axis is different from that of the stars. The core parameters in Figure 6 could result if NGC 1316 swallowed a disk galaxy with a normal bulge of $M_{B} \sim-20$ (Kormendy 1984). If star formation were enhanced by the merger, this could also explain the high central surface brightness (Fig. 6, upper left) and the small central mass-to-light ratio (Fig. 7). 


\subsection{Mass-To-Light Ratios}

The existence of a metallicity-luminosity relation for elliptical galaxies suggests that mass-to-light ratios $M / L$ should correlate with $L$. The parameter relations of $\S 4.1$ predict that $M / L \propto L^{0.18}$ (see also Kormendy 1982a, 1984). However, plots of $M / L$ values against $M_{B}$ have not in the past shown a correlation (Schechter 1980). This situation is unsatisfactory, since the transitive application of several parameter correlations with scatter is dangerous.

Figure 7 shows mass-to-light ratios for the present sample as a function of $M_{B}$. Here $M / L=9 \sigma^{2} / 2 \pi G I_{0} r_{c}$ is calculated using King's method (King and Minkowski 1972; Richstone and Tremaine 1986). The results are relatively insensitive to seeing corrections, since $I_{0} r_{c}$ varies only slowly with seeing (Schechter 1980). With more accurate core parameters, a correlation between $M / L_{V}$ and $M_{B}$ is clearly seen. For ellipticals, $M / L_{V} \propto L^{0.20 \pm 0.04}$, similar to the predicted relation and also to the estimate from the metallicity-luminosity relation. From the dependence of giantbranch stellar luminosity on metallicity, Tinsley (1978) derived $M / L_{B} \propto L_{B}^{0.13}$.

The mean mass-to-light ratio of old disks provides a welcome check of the present results. In our Galaxy, the disk mass is derived from the Oort-Bahcall analysis of the vertical dynamical equilibrium (Bahcall 1986, and references therein); in other galaxies it is based on a comparison of the vertical $\sigma$ in faceon galaxies with thicknesses of edge-on galaxies (van der Kruit and Freeman 1986). It is reassuring to see that old disks and ellipticals have similar $M / L$ ratios (Fig. 7 ).

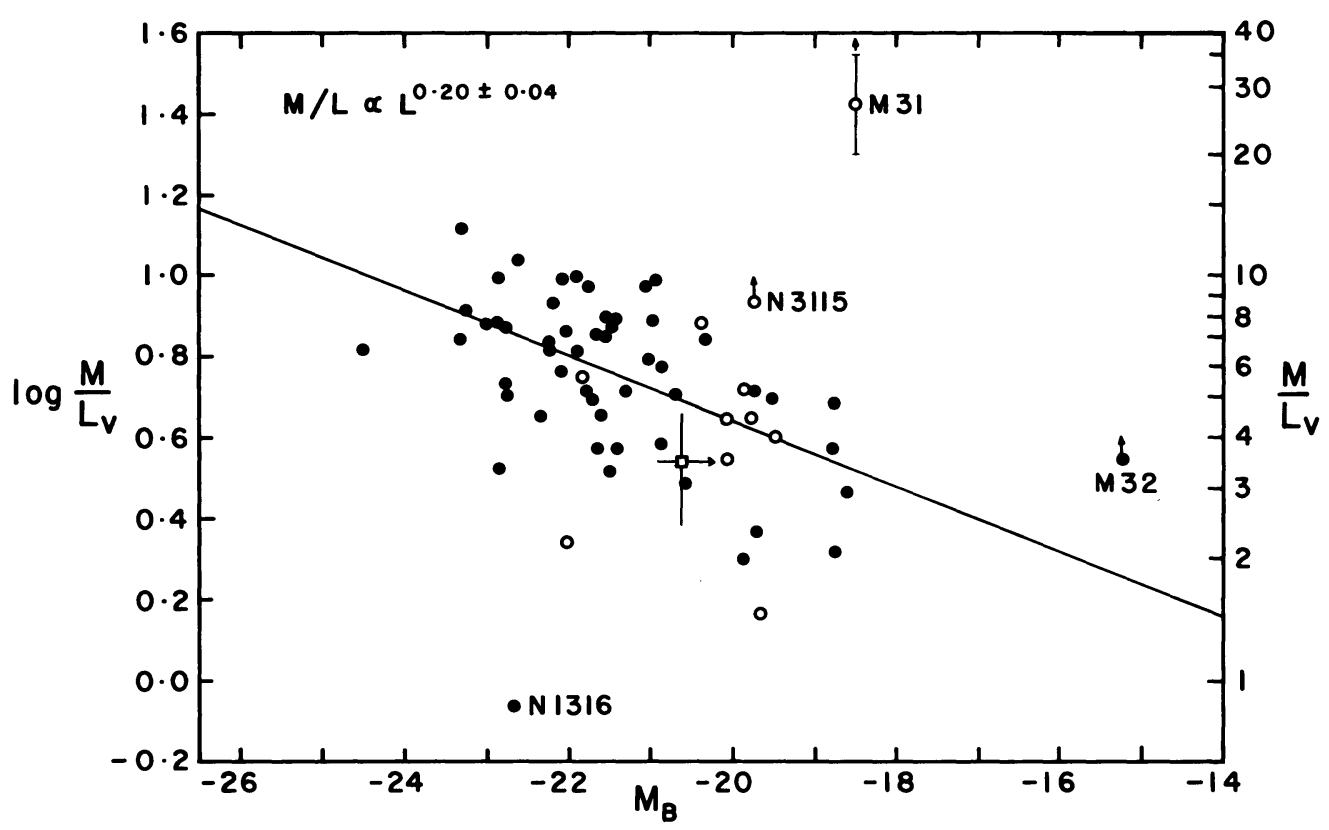

Fig. 7. - Core mass-to-light ratios for bulges and elliptical galaxies. The line is a least-squares fit to the ellipticals. The plus sign is the mean $M / L_{V}$ for old disks; the error bar is the dispersion of values seen. The point is plotted at the mean luminosity of the galaxies rather than that of the disks (hence the arrow). 
An especially interesting implication of Figure 7 follows if the $M / L-L$ correlation holds to galaxies as faint as M32. Richstone (1987) reports that dynamical models of M32 over a large radius range show that $M / L_{V}=2.5 \pm 0.1$. This is smaller than the nuclear value, and fits the observed correlation with $L$. The value is interesting because it is as small as mass-to-light ratios in gobular clusters (Illingworth 1976; Gunn and Griffin 1979; Meylan and Mayor 1986). Dynamical models of globular clusters can account for all of the observed mass with stars and stellar remnants that formed with relatively ordinary mass functions. Richstone's result shows that we need to find little or no dark matter in the inner parts of M32, perhaps not even the kind believed to exist in the galactic disk. The correlations of $M / L$ and metallicity with luminosity then suggest that there is little or no exotic dark matter in the central parts of other ellipticals. It is worth investigating again whether ordinary stellar populations of various metallicities can explain all of the mass in galaxy cores.

\section{MASSIVE BLACK HOLES IN GALAXY NUCLEI}

\subsection{Introduction}

The most interesting question we would like to ask of galaxy nuclei is whether they contain massive black holes (hereafter BHs). Compelling motivation is provided by a variety of nuclear activity - modest activity like that in our Galaxy, Seyfert nuclei, radio sources (especially radio continuum jets), and quasars. The activity is believed to originate through the accretion of gas by a nuclear "monster" (e. g., Rees 1984), but direct evidence for BHs is limited. The most conclusive case is the Galaxy, in which the nuclear radio source Sgr A has prodigious power but a size of $<20 \mathrm{AU}$ (Lo et al. 1985). For other galaxies I discuss only the evidence based on core dynamics. Published work has been limited by insufficient resolution and a general lack of information about cores. Before we knew that cores are anisotropic, it was easy to misinterpret effects seen in any one galaxy as resulting from a $\mathrm{BH}$. For example, Sargent et al. (1978) and Young et al. (1978) thought they had detected a $\mathrm{BH}$ in M87 because of a velocity dispersion profile and a nonisothermal brightness distribution that turn out to be just like those in other galaxies (Illingworth 1981; Lauer 1985b; Kormendy 1985a).

Motivated in part by the excellent seeing at the CFHT, I have begun a systematic search for nuclear BHs in early-type galaxies. A wide range of galaxies is being examined, including radio galaxies. However, because of the uncertainty in the velocity distributions of bright ellipticals, I put special emphasis on objects that rotate rapidly, i. e., faint ellipticals and bulges.

\section{$5.2 \mathrm{M} 31$}

The best evidence for a nuclear BH outside our Galaxy is in M31. Dressler (1984) saw an unresolved central jump in $V(r)$; Walker (1974) observed a maximum rotation velocity of $104 \mathrm{~km} \mathrm{~s}^{-1}$ at $r=2^{\prime \prime}$. Dressler also found an increase in $\sigma$ of $35 \%$ from $145 \mathrm{~km} \mathrm{~s}^{-1}$ to $196 \mathrm{~km} \mathrm{~s}^{-1}$ at the center. He concluded that the massto-light ratio increases inward by an order of magnitude; this could be explained by a nuclear $\mathrm{BH}$ of mass $\sim 10^{7} M_{\odot}$. Dressler's observations were obtained with a $2^{\prime \prime}$ slit, 0.58 pixels and a seeing of $2^{\prime \prime}$ FWHM. 


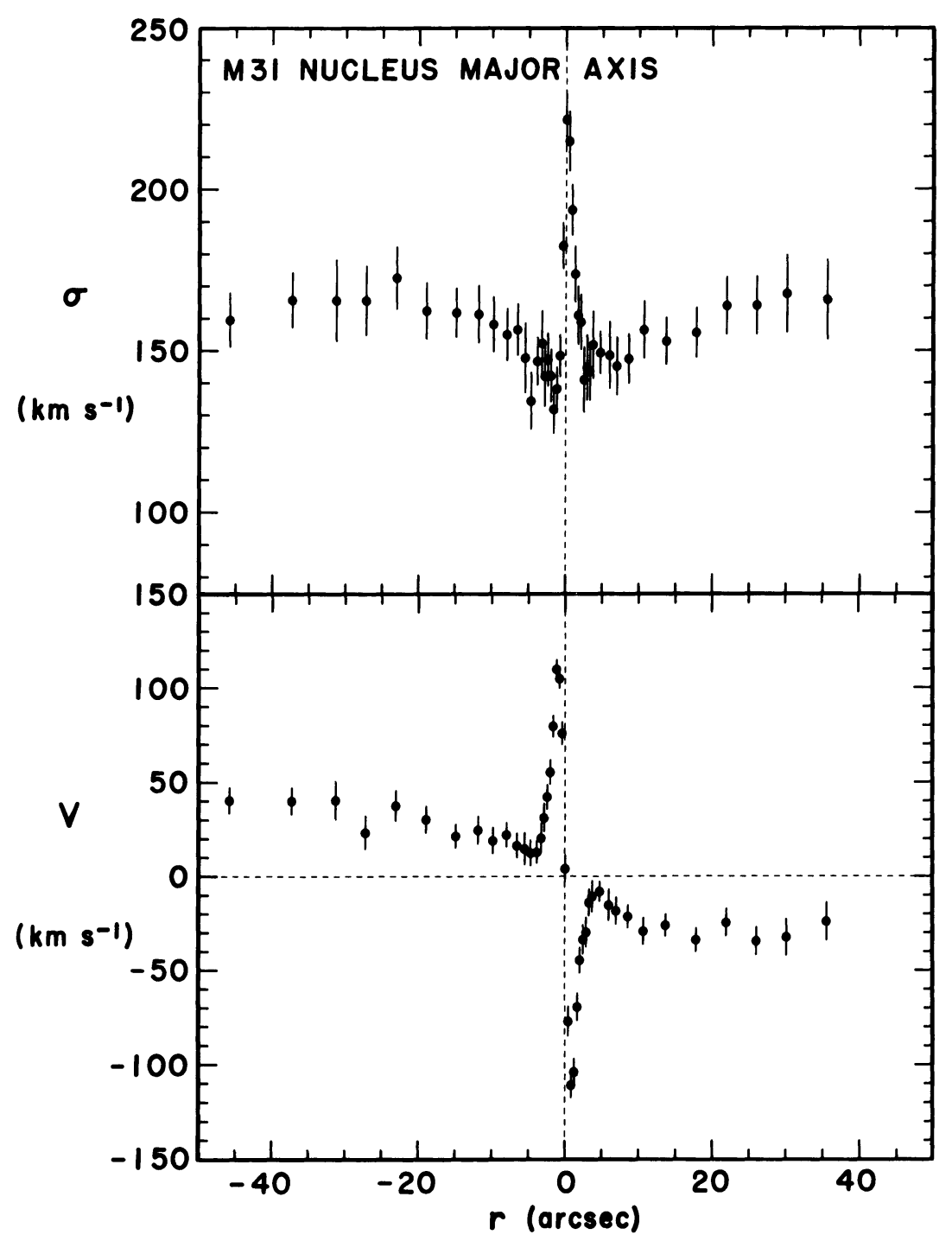

Fig. 8. - Rotation and dispersion profiles along the major axis of the nucleus of M31. Near the center, points are separated by 0.41 .

The present observations were obtained with a slit width of 0.5 , pixels of 0.41 and a seeing of 1.2 FWHM. Spectra were taken along the disk major axis, the major axis of the nucleus (26 $6^{\circ}$ from the major axis), and the minor axis. Figure 8 shows some of the results. The nucleus spins very rapidly. The central velocity gradient is still unresolved; the apparent peak velocity of $110 \mathrm{~km} \mathrm{~s}^{-1}$ at $r=11^{\prime \prime} 0$ is certainly an underestimate. The dispersion gradient is also unresolved; on one side $\sigma$ decreases in only 0.18 from $222 \mathrm{~km} \mathrm{~s}^{-1}$ to the value $145 \mathrm{~km} \mathrm{~s}^{-1}$ in the inner bulge. The asymmetry in the dispersion profile is real (cf. Dressler 1984); it may be due to dust absorption (Nieto et al. 1986). 


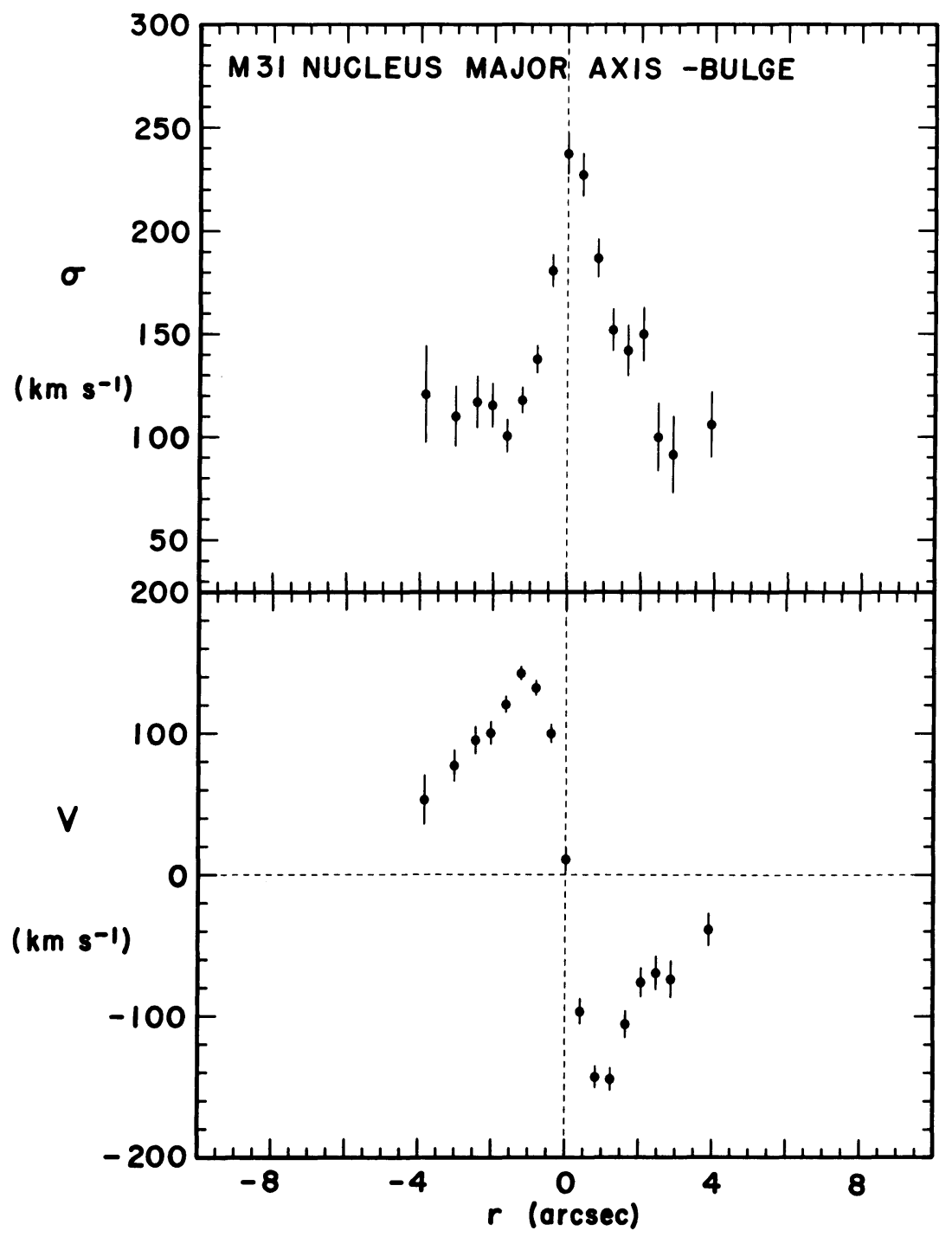

Fig. 9. - Rotation and dispersion profiles of the nucleus of M31 as recalculated after subtraction of the bulge spectrum.

Figure 8 shows that the nucleus is well differentiated from the bulge. In my spectra it outshines the bulge by a factor of 5 at the center; also, $r_{c, a p p}=17^{\prime \prime}$ for the bulge but only 1.11 for the nucleus. Tremaine and Ostriker (1982) have shown that the bulge and nucleus are, in fact, dynamically independent. I am therefore measuring two superposed but independent stellar populations: stars in the bulge certainly pass in front of and behind the nucleus. Moreover, the bulge has a smaller apparent velocity dispersion than the nucleus. The Fourier Quotient program reacts to such a mixture by weighting the cold component more highly than its relative contribution to the light (Whitmore 1980; McElroy 1983). To get an accurate 
measurement of the nucleus, I need to subtract the bulge spectrum before running the Fourier program. This is easy to do, since the relative light contributions are easy to determine from the spectrophotometry given the large ratio of bulge and nuclear core radii. The results are shown in Figure 9. As expected, the velocity gradients are larger than in Figure 8. The nucleus has a maximum observed rotation velocity of $143 \pm 5 \mathrm{~km} \mathrm{~s}^{-1}$ at $r=1{ }^{\prime \prime} 1$ and an apparent central velocity dispersion of $241 \pm 7 \mathrm{~km} \mathrm{~s}^{-1}$.

The surprise in Figure 9 is the dispersion at $r \gtrsim 2^{\prime \prime}$. Here $\sigma=107 \pm 5 \mathrm{~km} \mathrm{~s}^{-1}$, much smaller than in the bulge. This is clear from the spectra: the absorption lines $\mathrm{Mg}$ I $\lambda \lambda 5167$ and $5173 \AA$ are partly resolved in the outer nuclear spectrum but not in the total spectrum or in the bulge spectrum that was subtracted. Spectra along all three position angles show this behavior. The mean nuclear dispersion at $r>2^{\prime \prime}$ is $107 \pm 4 \mathrm{~km} \mathrm{~s}^{-1}$ (internal error). Given the large rotation velocity, this implies that the nucleus of MS1 is a disk. The "oblate line" describing isotropic rotating spheroids in the $V_{\max } / \sigma-\epsilon$ diagram would predict $V_{\max } / \sigma=0.8$ for the E4 shape of the nucleus; an inclined disk would show larger values. Distinct nuclei like that in MS1 may be produced from disk gas that falls into the center in triaxial potentials. Evidence for the importance of such gas flows is reviewed in Kormendy $(1982 a, b)$ and in Kormendy and Illingworth (1983). One additional piece of evidence: dust clearly knows how to find the center; it tends to settle in a ring or disk at $r \lesssim r_{c}$ (Kormendy and Stauffer 1987).

I can now estimate the central mass-to-light ratio. The precise value depends on how much of the central dispersion gradient is unresolved rotation. I consider two extreme cases. If all of the apparent dispersion gradient is unresolved rotation and $\sigma=107 \mathrm{~km} \mathrm{~s}^{-1}$ everywhere, then the shallowest possible rotation curve would have to rise to $260 \mathrm{~km} \mathrm{~s}^{-1}$ in the disk plane at $r \simeq 0$.' $^{\prime}=\mathrm{FWHM} / 2$. The corresponding central $M / L_{V}$ is $\sim 20$. Alternatively, if all of the dispersion gradient is real, then King's method corrected for rotation gives $M / L_{V}=35$. Since I have not yet made seeing corrections, these are lower limits. Similar values were derived previously (Light et al. 1974; Tremaine and Ostriker 1982). However, they are now more secure because of the improved kinematic resolution and because the rapid rotation eliminates the freedom to postulate large velocity anisotropies. Mass-to-light ratios of $20-35$ are much higher than values in other galaxies (Fig. 7). The unusually high dispersion is emphasized further in Figure 6 (lower left). There is therefore a strong case in M31 for a nuclear BH of mass $\sim 2 \times 10^{7} \mathrm{M}_{\odot}$.

\section{$5.3 \mathrm{M} 32$}

The second reported case of a nuclear BH is M32. Tonry (1984) and Dressler (1984) found an unresolved central jump in $V(r)$ to $\gtrsim 36 \mathrm{~km} \mathrm{~s}^{-1}$ and a $40 \%$ rise in $\sigma$ from 60 to $85 \mathrm{~km} \mathrm{~s}^{-1}$ at the center. Both authors concluded that $M / L$ rises toward the center; Tonry estimated a possible BH mass of $\sim 5 \times 10^{6} M_{\odot}$. With the CFHT it is again possible to make a significant improvement in resolution: for Tonry (1984), Dressler (1984) and the present work, seeing FWHM values are, respectively, $2^{\prime \prime} 5-3^{\prime \prime}, 2^{\prime \prime}$, and $1 . \prime 00 \pm 0$ !'05. Slit width $\times$ pixel size values are, respectively, $2^{\prime \prime} \times 0.58,2^{\prime \prime} \times 0.758$, and $0.4 \times 0.441$. 


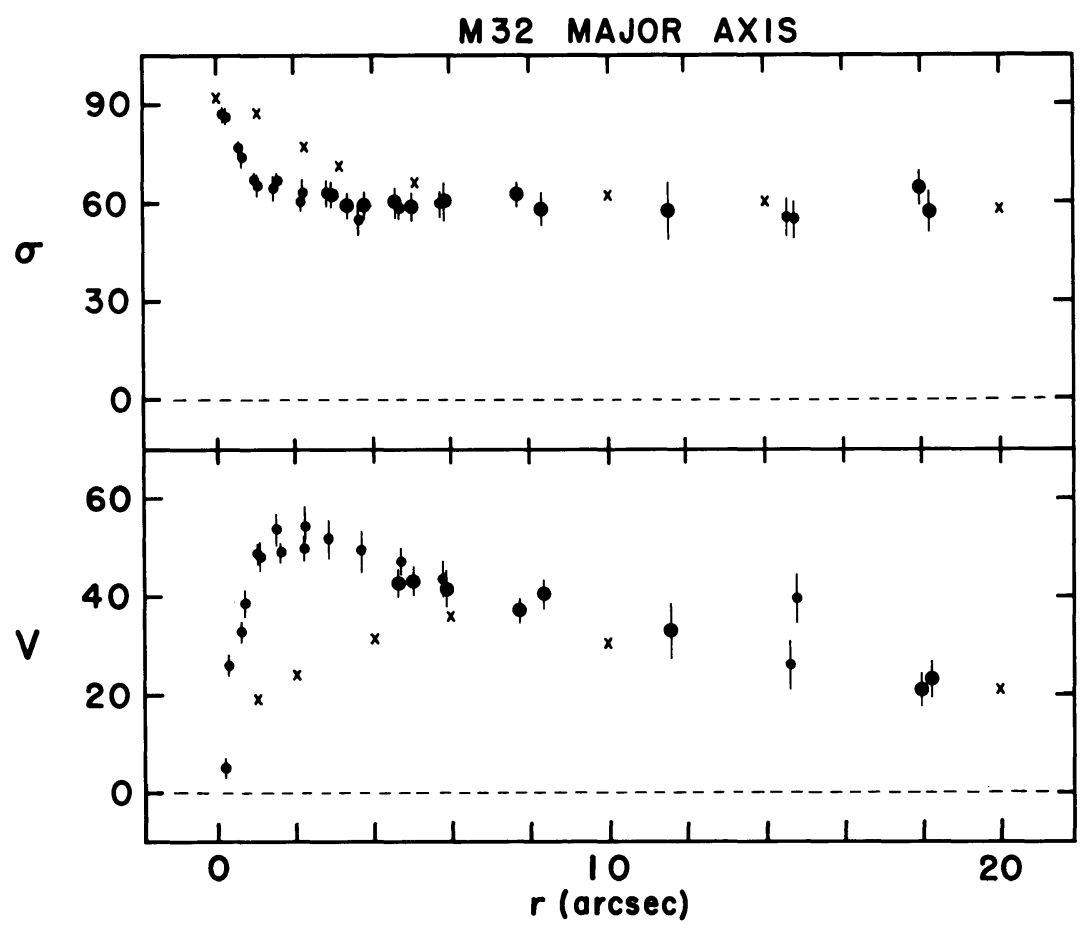

Fig. 10. - Rotation and velocity dispersion curves along the major axis of M32. The crosses show measurements by Tonry (1984); the velocities were taken at position angle $0^{\circ}\left(20^{\circ}\right.$ from the major axis) and the dispersions at position angle $90^{\circ}$. Tonry's "minor axis" dispersion measurements are shown because they were obtained in better seeing than his $\mathrm{PA}=0^{\circ}$ data.

The rotation and dispersion curves from the CFHT (Fig. 10) show that the $V$ and $\sigma$ gradients are much larger at small radii than in published measurements. Also, $V(r)$ reaches $>51 \mathrm{~km} \mathrm{~s}^{-1}$ at $r \simeq 2^{\prime \prime}$. But the central $\sigma=87 \mathrm{~km} \mathrm{~s}^{-1}$ is no larger than before, despite the improvement in resolution. All of the apparent dispersion gradient could be unresolved rotation. The formal core $M / L_{V}=4.1$ (corrected for rotation) is plotted in Figure 7. It is not very accurate because of the large seeing corrections, but it is also not especially large for the luminosity of the galaxy. The contrast between M31 and M32 is well illustrated in Figure 6: $r_{c}$ and $\mu_{0 V}$ are similar but $\sigma \geq 241 \mathrm{~km} \mathrm{~s}^{-1}$ in M31 and only $\sim 87 \mathrm{~km} \mathrm{~s}^{-1}$ in M32. Since $M / L \propto \sigma^{2}$, this is a large difference. Based on these results alone, I do not see strong evidence for a nuclear $\mathrm{BH}$.

Richstone (1987) points out that $M / L_{V}=2.5 \pm 0.1$ is closely constrained at larger radii. Therefore $M / L_{V}$ does rise toward the center. Based on his dynamical models, Richstone concludes that M32 remains an interesting $\mathrm{BH}$ candidate. 


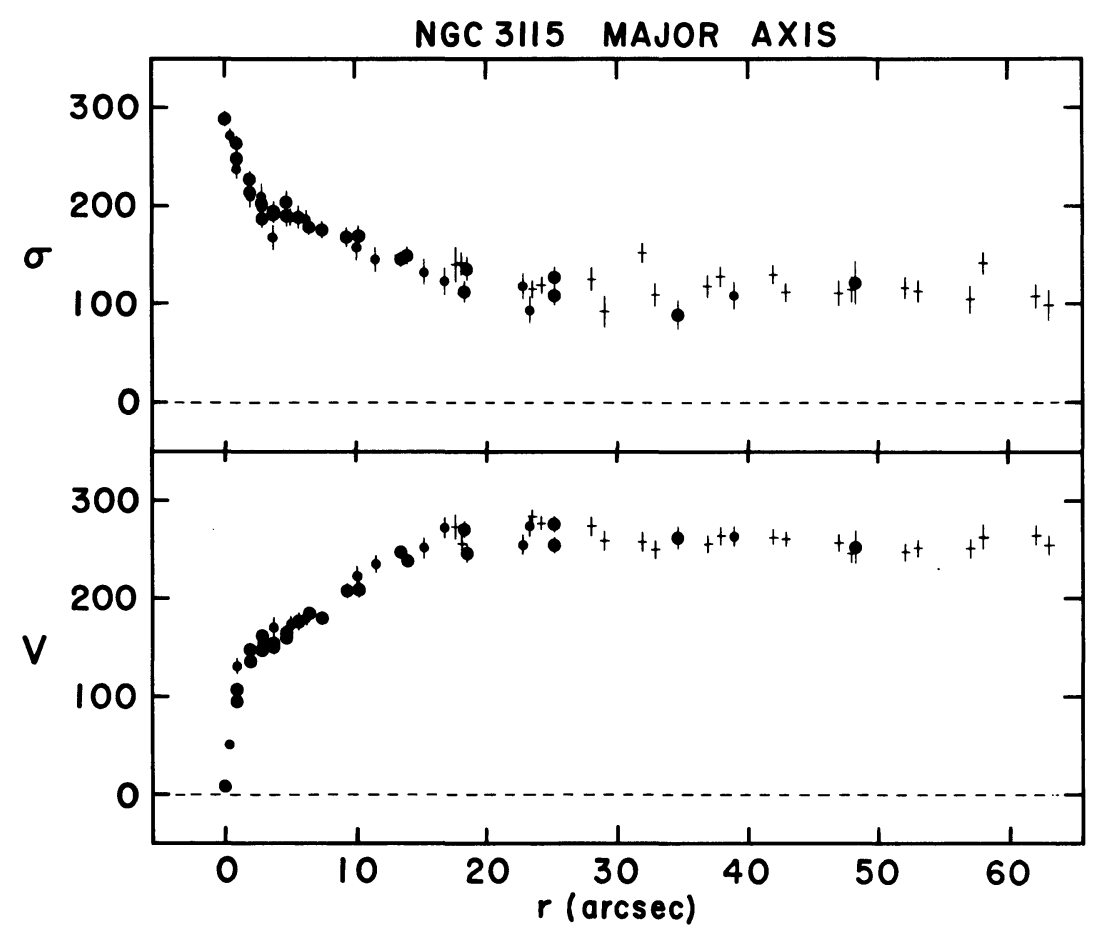

Fig. 11. - Rotation and velocity dispersion curves in NGC 3115. Large points are from an $1800 \mathrm{~s}$ exposure and small ones from a $900 \mathrm{~s}$ exposure with the CFHT. The plus signs are measurements by Illingworth and Schechter (1982).

\subsection{NGC 3115}

The present program has produced one new $\mathrm{BH}$ candidate. Figure 11 shows rotation and dispersion curves for the edge-on S0 galaxy NGC 3115. The slit width was $0 ! \prime 8$, the pixel size was $0 ! 92$, and the seeing was poor (FWHM = $1{ }^{\prime \prime} 7$ ). The rotation curve has an unresolved rise to $>120 \mathrm{~km} \mathrm{~s}^{-1}$ at $r=1^{\prime \prime}$. The velocity dispersion rises by $50 \%$ from $190 \mathrm{~km} \mathrm{~s}^{-1}$ at $r \simeq 3^{\prime \prime}$ to $288 \pm 8 \mathrm{~km} \mathrm{~s}^{-1}$ at the center. I assume that the velocity distribution is isotropic, because of the rapid rotation (Illingworth and Schechter 1982) and because my photometry shows that there is a nuclear disk at $r \leqslant 2^{\prime \prime}$. Then the central $M / L_{V}>8.6$. This is a lower limit, because I have not corrected for seeing. $M / L_{V}$ is somewhat large for the luminosity of the bulge (Fig. 7). Also, $\sigma$ is well outside the distribution of values for other galaxies (Fig. 6, lower left). This suggests the presence of a central mass concentration of $\sim 5 \times 10^{8} \mathrm{M}_{\odot}$. 


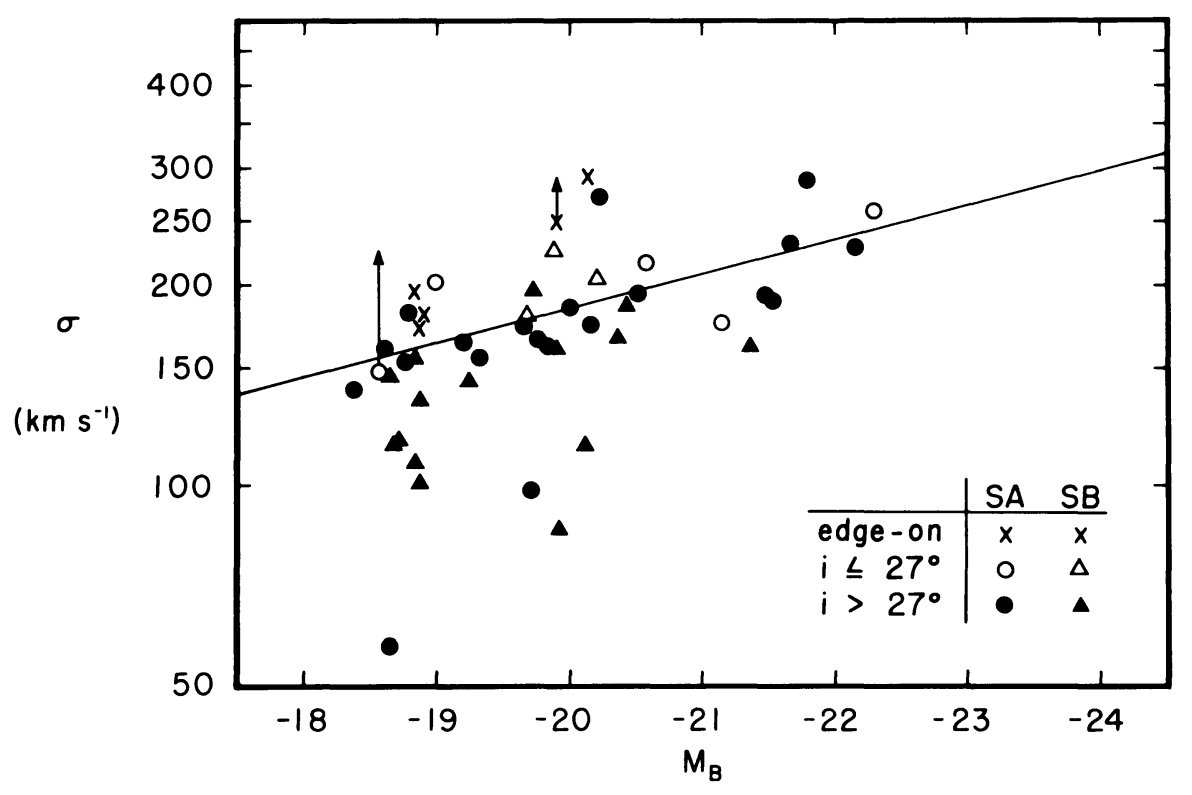

Fig. 12. - Faber-Jackson relation for bulges of various inclinations $\left(90^{\circ}-i\right)$. The solid line is the mean relation for SA0-bc galaxies. The arrows show the present measurements of M31 and NGC 3115 (before seeing corrections).

\subsection{Ubiquitous Spinning Nuclei and Central Black Holes?}

Figure 12 shows the Faber-Jackson relation for bulges of disk galaxies, from Kormendy and Illingworth (1983). We noted that more inclined bulges have larger $\sigma$. The zeropoint, say $\sigma$ at $M_{B}=-21$, is $208 \pm 8 \mathrm{~km} \mathrm{~s}^{-1}$ for SA0-bc galaxies and smaller for SB galaxies but $234 \pm 10 \mathrm{~km} \mathrm{~s}^{-1}$ for galaxies within $27^{\circ}$ of edge-on. We had no obvious explanation. We noted that the observations could be due to rapid central rotation produced by nuclear mass concentrations, but we didn't really believe it at the time. Given the results of the previous sections, it is interesting to wonder whether rapidly spinning nuclear disks and central mass concentrations are widespread phenomena in galaxy bulges. The search with the CFHT for BHs in galaxy nuclei is continuing.

\section{REFERENCES}

Bahcall, J. N. 1986, in IAU Symposium 117, Dark Matter in the Universe, ed. J. Kormendy and G. R. Knapp (Dordrecht: Reidel), p. 17.

Bendinelli, O., Parmeggiani, G., and Zavatti, F. 1984, Astr. Ap., 140, 174.

Binney, J. 1976, M. N.R. A. S., 177, 19.

Binney, J. 1978, M. N. R. A. S., 183, 501.

Binney, J., and Mamon, G. A. 1982, M. N. R. A. S., 200, 361.

Carlberg, R. 1987, in IAU Symposium 127, Structure and Dynamics of Elliptical Galaxies, ed. T. de Zeeuw (Dordrecht: Reidel), p. 353.

Dressler, A. 1984, Ap. J., 286, 97. 
Duncan, M. J., and Wheeler, J. C. 1980, Ap. J. (Letters), 237, L27.

Faber, S. M., and Jackson, R. E. 1976, Ap. J., 204, 668.

Gunn, J. E., and Griffin, R. F. 1979, A. J., 84, 752.

Illingworth, G. 1976, Ap. J., 204, 73.

Illingworth, G. 1977, Ap. J. (Letters), $218, \mathrm{~L} 43$.

Illingworth, G. 1981, in The Structure and Evolution of Normal Galaxies, ed.

S. M. Fall and D. Lynden-Bell (Cambridge: Cambridge Univ. Press), p. 27.

Illingworth, G., and Schechter, P. L. 1982, Ap. J., 256, 481.

Kent, S. M. 1983, Ap. J, 266, 562.

King, I. R. 1966, A. J., 71, 64.

King, I. 1978, Ap. J., $222,1$.

King, I. R., and Minkowski, R. 1972, in IAU Symposium 44, External Galaxies and Quasi-Stellar Objects, ed. D. S. Evans (Dordrecht: Reidel), p. 87.

Kormendy, J. 1982a, in Morphology and Dynamics of Galaxies, ed. L. Martinet and M. Mayor (Sauverny: Geneva Observatory), p. 113.

Kormendy, J. 1982 b, Ap. J., 257, 75.

Kormendy, J. 1984, Ap. J., 287, 577.

Kormendy, J. 1985a, Ap. J. (Letters), 292, L9.

Kormendy, J. 1985b, Ap. J., 295, 73.

Kormendy, J. 1986, in Nearly Normal Galaxies: From the Planck Time to the Present, ed. S. M. Faber (New York: Springer-Verlag), in press.

Kormendy, J., and Illingworth, G. 1983, Ap. J., 265, 632.

Kormendy, J., and Stauffer, J. 1987, in IAU Symp. 127, Structure and Dynamics of Elliptical Galaxies, ed. T. de Zeeuw (Dordrecht: Reidel), p. 405.

Lauer, T. R. 1985a, Ap. J. Suppl., 57, 473.

Lauer, T. R. $1985 b, A p$. J., $292,104$.

Light, E. S., Danielson, R. E., and Schwarzschild, M. 1974, Ap. J, 194, 257.

Lo, K. Y., Backer, D. C., Ekers, R. D., Kellermann, K. I., Reid, M., and Moran, J. M. 1985, Nature, 315, 124.

McElroy, D. B. 1983, Ap. J., 270, 485.

Meylan, G., and Mayor, M. 1986, Astr. Ap., 166, 122.

Nieto, J.-L., Macchetto, F. D., Perryman, M. A. C., di Serego Alighieri, S., and Lelièvre, G. 1986, Astr. Ap., 165, 189.

Rees, M. J. 1984, Ann. Rev. Astr. Ap., $22,471$.

Richstone, D. O. 1987, in IAU Symposium 127, Structure and Dynamics of Elliptical Galaxies, ed. T. de Zeeuw (Dordrecht: Reidel), p. 261.

Richstone, D. O., and Tremaine, S. 1985, Ap. J., 296, 370.

Richstone, D. O., and Tremaine, S. 1986, A. J., 92, 72.

Sargent, W. L. W., Young, P. J., Boksenberg, A., Shortridge, K., Lynds, C. R., and Hartwick, F. D. A. 1978, Ap. J., $221,731$.

Schechter, P. L. 1980, A. J., 85, 801.

Schweizer, F. 1979, Ap. J., 233, 23.

Schweizer, F. 1980, Ap. J., 237, 303.

Schweizer, F. 1981, A. J., 86, 662.

Tinsley, B. M. 1978, Ap. J., $222,14$.

Tonry, J. L. 1984, Ap. J. (Letters), 283, L27.

Tremaine, S. D., and Ostriker, J. P. 1982, Ap. J., 256, 435.

van der Kruit, P. C., and Freeman, K. C. 1986, Ap. J., 303, 556.

Walker, M. F. 1974. Publ. A. S. P., 86, 861.

Whitmore, B. C. 1980, Ap. J., $242,53$.

Young, P. J., Westphal, J. A., Kristian, J., Wilson, C. P., and Landauer, F. P. 1978, Ap. J., 221, 721. 


\section{DISCUSSION}

Lupton: Using your correlation, at what $M_{B}$ is the central two-body relaxation time equal to the Hubble time?

Kormendy: Fifty-five galaxies with $-18.6 \geq M_{B} \geq-24.5$ give $t_{\text {or }} \propto L^{2.3 \pm 0.1}$ with $t_{\text {or }}=10^{10} \mathrm{yr}$ at $M_{B}=-17.4$ (assuming a Hubble constant $H_{0}=50 \mathrm{~km} \mathrm{~s}^{-1} \mathrm{Mpc}^{-1}$ ). However, the smallest 14 values of $t_{o r}$ belong to galaxies that are very poorly resolved. Since $t_{\text {or }} \propto \sigma r_{c}^{2}$, and seeing corrections are usually underestimated, I expect that these values of $t_{\text {or }}$ are upper limits, that the real relation may be slightly steaper than I have found, and that $t_{\text {or }}$ may already equal $10^{10} \mathrm{yr}$ at an absolute magnitude brighter than $\mathbf{- 1 7 . 4}$.

King: You seem to have determined a core radius for M32, yet I remember that the unpublished Stratoscope curves, which I have seen, have a much smaller core radius - if any at all. How did you determine your core radius for M32? This is important, because the derived $M / L$ depends linearly on the $r_{c}$ assumed (since it is $I_{0} r_{c}$ that goes into the formula, while $I_{0} r_{c}^{2}$ is conserved).

Kormendy: The value I quoted, $r_{c} \sim 1.3 \mathrm{pc}$, is a lower limit based on a profile that was very poorly resolved $\left(r_{c, a p p} / \sigma_{*}<3\right)$. I applied the best correction for seeing that I could, using the method discussed in the text, but I would not be surprised if any real core were much smaller than I derived.

Gunn: If you interpret your M31 data as due to motion in a central disk, is the fall of rotation velocity with radius consistent with a Keplerian potential?

Kormendy: Formally the best fit is $v \propto r^{-1}$, but $v \propto r^{-1 / 2}$ is not excluded by the rather large error bars.

Porter: You said that the different central values of the distribution function in ellipticals and disks argues against formation of ellipticals by dissipationless mergers of disks-but would you expect disks to merge without dissipation? Or do you think the differences in $f_{0}$ are large enough to argue against mergers anyway?

Kormendy: This question should really be addressed to Ray Carlberg, whose work I was summarizing. As I understand it, the argument excludes only dissipationless mergers of stellar disks. Any gas in two merging disks could produce high phasespace densities in stars that they form. Also, mergers of galaxies that already contain bulges with high central densities could easily make cores like those we see in ellipticals.

Gerhard: Do you confirm the 2:1 apparent axial ratio of the nucleus of M31, as found in the Stratoscope II observations (Light, Danielson and Schwarzschild, 1974, Astrophys. J., 194, 257), which would-in the disk interpretation of the nuclear rotation-increase the velocities correspondingly?

Nieto: About the nucleus of M31, I would like to comment on the observations made in the UV at the CFH Telescope with the ESA Photon-Counting-Detector, which is the scientific prototype of the Faint Object Camera of ST. The resolution (FWHM) 
was 0.65 arcseconds. We found indeed a dust lane in the nucleus oriented along the major axis, i.e., perpendicular to the rotation axis found in 1960 by Lallemand, Duchesne \& Walker (Publ. Astr. Soc. Pacific, 72, 76); it is suggested that the nucleus was close to an oblate system. In addition, we found a strong UV excess by comparing our profile with the radial profile obtained by Kent. This result should be however taken with care since it comes from two different instruments, and should be confirmed. (Nieto, Macchetto, Perryman, di Serego Alighieri \& Lelièvre, 1986, Astr. Astrophys., 165, 189). These observations were made in the Time Resolved Imaging Mode of the PCD. We are presently applying image centering and - selection algorithms in order to improve the resolution of these data and to go down to the sub-half arc second level. We may have therefore a few more things to say soon.

Kormendy: These beautiful observations appear to support the hypothesis that the nucleus was made out of infalling disk gas; they show that the interstellar matter reaches all the way in to the nucleus, and suggest that young stars are present.

Sussman: Assuming M31 is $750 \mathrm{kpc}$ distant, 1 arcsec projects to about 4 pc. The nucleus thus has a radius of a few pc, from your measurements. Your dynamical data requires that we stuff a few times $10^{7} M_{\odot}$ into that radius. This density does not seem terribly inconsistent with a large, purely stellar cluster. Is there strong evidence that I miss here, indicating that the mass is concentrated into an unusual compact object? Is this conclusion supported by the dynamical data alone?

Kormendy: At the luminosity of the bulge of $\mathrm{M} 31, M / L_{V}$ values for other bulges and ellipticals lie between $\sim 2$ and $\sim 6$. The bulge of M31 itself has a normal $M / L_{V}$. But the mass-to-light ratio of the nucleus, calculated in the same way and of course taking into account the very high surface brightness, is large. At $M / L_{V}=20-40$ (even without seeing corrections) it is far outside the distribution of $M / L_{V}$ values for other stellar systems which have similar $\mathrm{K}$-star spectra. Of course, it is always possible to explain such a mass-to-light ratio with a sufficiently strange stellar mass function, but the resulting stellar population would be very different from any that we have seen elsewhere. In fact, if the blue central colors measured by Nieto et al. are confirmed, the indications are, if anything, that the mass-to-light ratio of the stars in the nucleus is lower, rather than higher, than average. 